\title{
The incidence and effect of coagulopathy on short-term outcomes in patients undergoing craniotomy following traumatic brain injury
}

\author{
Sonia Bansal, Rohini M. Surve, Madhusudhan B. Rao', Bhadri V. Narayan, Mariamma Philip², \\ S. Sampath
}

\begin{abstract}
Background: Coagulopathy in isolated traumatic brain injury (TBI) is well-known, and studies have found an association between coagulopathy and unfavourable outcomes. This study was conducted to determine the incidence and causes of coagulopathy in patients with TBI undergoing craniotomy and its effect on post-operative outcome. Materials and Methods: The data collected was demographics, computed tomography diagnosis, post-resuscitation Glasgow Coma Scale (GCS) score, pre- and post-operative platelet count, liver function tests, intraoperative blood loss and transfusion, fluids infused and incidence of redo surgery. Point of care (Coaguchek XS) monitor was used to obtain prothrombin time and international normalised ratio (INR) at $24 \mathrm{~h}$ and $72 \mathrm{~h}$ of injury. Coagulopathy was defined as INR $\geq 1.3$ and thrombocytopenia as platelet count $\leq 100,000 / \mathrm{mcL}$. Outcome measures assessed were the length of hospital stay, GCS at discharge and in-hospital mortality. Results: In 166 patients, the average pre-operative GCS was $8.8 \pm 3.6$. The incidence of coagulopathy was $42.8 \%$ and increased to $55.6 \%$ on the $3^{\text {rd }}$ day, and thrombocytopenia from $3.5 \%$ in the first $24 \mathrm{~h}$ increased to $14.7 \%$ at $72 \mathrm{~h}$. Patients with coagulopathy had lower pre-operative admission GCS (median 7 vs. 9, $P=0.03$ ), greater intraoperative blood loss and received more intravenous fluids. There was no difference in the incidence of post-operative haematomas, length of hospital stay and GCS at discharge or mortality. Conclusion: In patients with TBI, the incidence of coagulopathy increased at the end of $72 \mathrm{~h}$. In this study, there was no difference in outcomes in patients who underwent craniotomy with deranged coagulation.
\end{abstract}

Key words: Coagulopathy, post-operative outcome, traumatic brain injury

Departments of Neuroanaesthesia, ${ }^{2}$ Biostatistics and ${ }^{3}$ Neurosurgery, National Institute of Mental Health and Neurosciences, Bengaluru, Karnataka, ${ }^{1}$ Department of Neuroanaesthesia and Neuroritical Care, Muthoot Health Care Limited, Kozhencherry, Pathanamthitta, Kerala, India

Address for correspondence:

Dr. Bhadri V. Narayan, Department of Neuroanaesthesia and

Neuroritical Care, $3^{\text {rd }}$ Floor, Faculty Block, Neurocentre,

National Institute of Mental Health and Neurosciences,

Bengaluru - 560 029, Karnataka, India.

E-mail: n_bhadri@yahoo.com

\begin{tabular}{|l|l|}
\hline \multicolumn{2}{|c|}{ Access this article online } \\
\hline Quick Response Code: & Website: \\
\hline & www.jnaccjournal.org \\
\cline { 2 - 2 } & \\
\hline & \\
\hline
\end{tabular}

\section{INTRODUCTION}

Normal haemostasis is very important in patients with traumatic brain injury (TBI) to prevent progression of microhaemorrhages into larger haematomas. Coagulation abnormalities following TBI can be due to cerebral tissue factor release $\mathrm{e}^{[1]}$ and multiple other factors. Coagulopathy as a consequence of TBI can itself act as a secondary insult. Coagulation abnormalities comprise

This is an open access article distributed under the terms of the Creative Commons Attribution-NonCommercial-ShareAlike 3.0 License, which allows others to remix, tweak, and build upon the work non-commercially, as long as the author is credited and the new creations are licensed under the identical terms.

For reprints contact: reprints@medknow.com

How to cite this article: Bansal S, Surve RM, Rao MB, Narayan BV, Philip M, Sampath S. The incidence and effect of coagulopathy on short-term outcomes in patients undergoing craniotomy following traumatic brain injury. J Neuroanaesthesiol Crit Care 2017;4:85-90. 
disturbances in coagulation leading to bleeding as well as hypercoagulability. ${ }^{[2]}$ Hypercoagulability can lead to disseminated intravascular coagulation or localised thrombotic occlusion of microvessels around the injured area leading to ischaemia. These disturbances have been shown to be associated with unfavourable outcomes. ${ }^{[3]}$ Early detection and prompt treatment of coagulopathy might improve outcomes in TBI. In this study, the primary aim was to determine the incidence of coagulopathy and its effect on intraoperative complications, on the incidence of post-operative haematomas and short-term post-operative outcome in a subpopulation of TBI patients presenting for an emergency craniotomy. The secondary aim was to determine the probable causes of this coagulopathy in patients with TBI.

\section{MATERIALS AND METHODS}

This was a prospective study conducted over a period of 1 year. Consecutive patients with isolated TBI, aged between 16 and 70 years admitted to the emergency department of our institute, who underwent craniotomy within $24 \mathrm{~h}$ of trauma were included in the study. Patients underwent craniotomy, for evacuation of either extradural haematoma $(\mathrm{EDH})$, subdural haematoma (SDH) or contusion or for the elevation of depressed fracture. Patients on antiplatelet agents or anticoagulants were excluded from the study. Furthermore, patients with polytrauma were excluded from the study as our centre caters to patients with only neurological injuries. Patients with polytrauma are referred to centres with respective facilities after providing basic resuscitation and care. Institute Ethics Committee approval was obtained for the study. The study was funded by Indian Society of Neuroanaesthesiology and Critical Care.

Our institute is a tertiary care centre for neurological patients. Patients with TBI are first triaged in emergency department where basic resuscitation (including intubation) and diagnostic workup including computed tomography $(\mathrm{CT})$ scan is done. The patient is transferred to emergency operation room (OR) for surgery or to observation ward as decided based on neurological assessment and CT scan findings. In this study, only patients who required surgery were included.

The following data were collected for all patients preoperatively: age, gender, mode of injury, time of injury as declared by the person who brought the patient, post-resuscitation Glasgow Coma Scale (GCS) score, pre-operative status of pupils, CT diagnosis, pre-operative haemoglobin, platelet count, serum electrolytes and renal and liver function tests.

In the OR, anaesthesia was induced as per the choice of the incharge anaesthesiologist. Coagulation testing was performed after induction of anaesthesia by the investigator and before the start of surgery. Prothrombin time (PT) and international normalised ratio (INR) was checked using point of care test (Coaguchek XS, Roche Diagnostic GmbH, Mannheim, Germany). This is a handheld monitor, and the test requires a drop of blood from the patient on a strip and the test result is displayed within a few seconds. It is a test that can be done at the bedside.

The results of coagulation test were not informed to the treating anaesthesiologist and the management of individual case was left to the discretion of the anaesthesiologist attending to the case. All patients underwent craniotomy. Intra-operative blood loss, crystalloids and colloids infused, blood and blood components transfused were recorded. Postoperatively, the patient was followed up for haemoglobin and platelet count and any repeat surgery for post-operative haematomas. The note was made of any blood or blood product transfusion. Repeat INR and PT were done after $72 \mathrm{~h}$ of injury. The coagulation testing was planned at only two-time points for all the patients irrespective of the result of the test. The patients were followed up till discharge. Outcome measures assessed were the length of hospital stay, GCS at discharge and in-hospital mortality.

Coagulopathy in our study was defined as INR $\geq 1.3$ and thrombocytopenia as platelet count $<100,000 / \mathrm{mcL}$. The cut-off for treating post-operative thrombocytopenia was kept at 50,000/mcL along with any clinical or radiological evidence of a bleed.

\section{Statistical analysis}

Continuous variables were described using mean and standard deviation and categorical variables using numbers and percentages. The two groups, that is, patients with and without coagulopathy were compared using $t$-test or Mann-Whitney U-test for the continuous variables and Chi-square test for categorical variables. Age was compared using independent sample $t$-test, all other continuous parameters were analysed using Mann-Whitney as they did not follow normal distribution. Binary logistic regression was used to find out predictors of deranged INR. The incidence of pre-operative derangement in coagulation was compared with post-operative coagulation using McNemar's test. The power of the study was $70 \%$.

\section{RESULTS}

A total of 166 patients were included in the study. The demographic characteristics of our study population are shown in Table 1 . The mean age, mean pre-operative GCS and mean duration between trauma and time of surgery were 36.6 (SD 13) years, 8.8 (SD 3.6) and 9.8 (SD 5.1) hours respectively. The most common single diagnosis was 
Table 1: Demographic characteristics of the patient population $(n=166)$

\begin{tabular}{|c|c|}
\hline Variable & Mean (SD) \\
\hline Age (years) & $36.6(13)$ \\
\hline Time between trauma and surgery (h) & $9.8(5.1)$ \\
\hline Pre-operative GCS & $8.8(3.6)$ \\
\hline Male:female (\%) & $82.5: 17.5$ \\
\hline \multicolumn{2}{|l|}{ CT diagnosis (percentage of patients) } \\
\hline Contusion & 12 \\
\hline Depressed fracture & 1.8 \\
\hline $\mathrm{EDH}$ & 27 \\
\hline Parenchymal haematoma & 1.2 \\
\hline $\mathrm{SDH}$ & 16.3 \\
\hline Combined & 42 \\
\hline \multicolumn{2}{|l|}{ Mode of injury (percentage of patients) } \\
\hline RTA & 66.3 \\
\hline Fall & 23 \\
\hline Assault & 6.6 \\
\hline Miscellaneous & 1.8 \\
\hline Not known & 2.4 \\
\hline $\begin{array}{l}\text { Influence of alcohol (yes:no) } \\
\text { percentage of patients }\end{array}$ & $29: 71$ \\
\hline \multicolumn{2}{|l|}{$\begin{array}{l}\text { Severity of head injury (percentage of } \\
\text { patients) }\end{array}$} \\
\hline Mild & 21 \\
\hline Moderate & 29 \\
\hline Severe & 50 \\
\hline \multicolumn{2}{|l|}{ Discharge GCS (percentage of patients) } \\
\hline $13-15$ & 64.5 \\
\hline $9-12$ & 14.5 \\
\hline $3-8$ & 11 \\
\hline Expired & 10 \\
\hline $\begin{array}{l}\text { Pre-operative thrombocytopenia } \\
\text { (yes:no) percentage of patients }\end{array}$ & $3.5: 96.5$ \\
\hline
\end{tabular}

EDH in $27 \%$ patients, followed by SDH in $16 \%$, however, $42 \%$ patients had more than one type of injury. The most common mode of injury was road traffic accident in $66 \%$ patients. Fifty per cent of the patients had a pre-operative GCS <8; and $64.5 \%$ patients had GCS $>13$ at discharge. The in-hospital mortality rate in this study was $10 \%$.

All patients were haemodynamically stable at presentation to OR. After induction, baseline temperature using oropharyngeal temperature probe was recorded. None of the patients had hypothermia defined as temperature $<36^{\circ} \mathrm{C}$. Intraoperatively, warming blanket was placed (Bair Hugger, Arizant Healthcare Inc., St Eden Prairie) and continuous temperature monitoring was done for all the patients as a standard of care at our institute.

The incidence of pre-operative thrombocytopenia in our study was $3.5 \%$ on the $1^{\text {st }}$ day which increased to $14.7 \%$ on the $3^{\text {rd }}$ day post-injury. Using an INR of $\geq 1.3$ as a cut-off to define coagulopathy, $42.8 \%$ patients (95\% confidence interval [CI]: 0.352-0.504) had deranged coagulation preoperatively.

Comparing the group of patients with or without deranged coagulation, there was no difference between the groups with regard to age, sex, diagnosis on CT scan, number of patients under the influence of alcohol at the time of injury and pre- and post-operative thrombocytopenia. The pre-operative GCS was significantly different between the two groups (7 vs. 9) with $P$ value of 0.029 . Patients with coagulopathy had PT $>14 \mathrm{~s}$ and this difference was also significantly different between the two groups $(98.6 \%$ vs. $29.8 \%$, $P<0.001)$. There was no difference between the groups with respect to liver enzymes except serum bilirubin, which was more in patients with deranged coagulation $(P=0.015)$.

Intraoperatively, patients with coagulation abnormality received more crystalloids, colloids and had more blood loss. The colloid used was $6 \%$ hydroxyethyl starch $130 / 0.4$ in $0.9 \%$ normal saline. There was a trend towards increased transfusion of fresh frozen plasma, platelets and lower discharge GCS in patients with deranged coagulation. However, no difference was found with the amount of blood transfusion, incidence of redo surgery for post-operative haematomas or length of hospital stay [Table 2].

Among the pre-operative factors which could predict deranged coagulation, only GCS turned out to be a significant variable. Logistic regression was used to find out the predictability of GCS for deranged coagulation. GCS had a significant $P$ value of 0.033 with Odd's ratio of 0.907 (95\% CI: 0.829-0.992), but the value of $R^{2}$ was low (0.038).

Post-operative INR was available in only 144 patients as some patients were discharged unexpectedly due to logistic reasons. On analysing the pre- and post-operative INR in these patients, it was found that 40 patients $(27.8 \%)$ who had normal INR pre-operatively, developed coagulation abnormalities postoperatively ( $3^{\text {rd }}$ day following trauma). On the other hand, 22 patients $(15.3 \%)$ who had deranged coagulation in the first $24 \mathrm{~h}$ had normal coagulation at $3^{\text {rd }}$ day post-injury. The incidence of coagulopathy 
increased to $55.6 \%$ by the $3^{\text {rd }}$ day which was statistically significant $(P=0.03)$ as shown in Table 3 .

\section{DISCUSSION}

In a study by Epstein et al., the authors aimed at determining the clinically significant cut-off value of INR which was associated with higher mortality. It was a retrospective study, conducted in 1718 patients with isolated TBI with the primary outcome being in-hospital mortality. The authors observed a sharp increase in mortality in patients with an INR $\geq 1.3$, which remained high, independent of injury severity score (ISS) or increased INR. Coagulopathic patients were eight times more likely to die before discharge (odds ratio: 8.2; 95\% CI: 5.6-12.0). Based on this study, we chose a cut-off of INR of $\geq 1.3$ to define coagulopathy in our study. ${ }^{[4]}$

Coagulopathy is a form of secondary injury and contributes to increased morbidity and mortality. However, its incidence varies in different studies because of the diversity in the defining criteria. Based on cut-off of INR $\geq 1.3$, the incidence of coagulopathy in our study in the Indian population with TBI presenting for emergency surgery was $42.8 \%$, and it increased to $55.6 \%$ on the $3^{\text {rd }}$ day after trauma. According to a meta-analysis of 34 studies by Harhangi et al. ${ }^{[2]}$ the overall frequency of coagulopathy is $32.7 \%$ (range $10 \%-97.5 \%$ ).

Table 2: Differential characteristics of patients with and without coagulopathy

\begin{tabular}{|c|c|c|c|c|}
\hline Variable & INR $<1.3(n=95)$ & INR $\geq 1.3(n=71)$ & Test statistic & $P$ \\
\hline Age in years, mean (SD) & $37(12.5)$ & $36(13.7)$ & $t=0.509$ & 0.611 \\
\hline Pre-operative GCS (median, range) & $9(3-15)$ & $7(4-15)$ & $U=2708.5$ & $0.029 *$ \\
\hline Male:female $(n)$ & $75: 20$ & $62: 8$ & $\chi^{2}=1.977$ & 0.160 \\
\hline CT diagnosis & & & & 0.155 \\
\hline Influence of alcohol, yes:no $(n)$ & 29:66 & 19:52 & $\chi^{2}=0.28$ & 0.596 \\
\hline Pre-operative thrombocytopenia, yes:no $(n)$ & $4: 80$ & 1:59 & $\chi^{2}=1$ & 0.317 \\
\hline Post-operative thrombocytopenia, yes:no $(n)$ & 11:77 & $12: 56$ & $\chi^{2}=0.808$ & 0.369 \\
\hline Pre-operative PT>14 s, yes:no $(n)$ & 28:66 & $69: 1$ & $\chi^{2}=78.56$ & $0.001^{*}$ \\
\hline Post-operative PT>14 s, yes:no (n) & $62: 19$ & 49:12 & $\chi^{2}=0.3$ & 0.589 \\
\hline Post-operative APTT>40, yes:no $(n)$ & 2:65 & $5: 43$ & $\chi^{2}=2.7$ & 0.100 \\
\hline SGOT>200, yes:no $(n)$ & 2:93 & $4: 66$ & $\chi^{2}=1.5$ & 0.221 \\
\hline SGPT>200, yes:no $(n)$ & 0:95 & $2: 68$ & $\chi^{2}=2.74$ & 0.097 \\
\hline Bilirubin >1.5 mg/dl, yes:no $(n)$ & $10: 84$ & $17: 51$ & $\chi^{2}=5.86$ & $0.015^{*}$ \\
\hline Redo surgery, yes:no $(n)$ & $3: 92$ & $2: 69$ & $\chi^{2}=0.16$ & 0.899 \\
\hline Intraoperative crystalloids (ml), median (range) & $2500(1000-5500)$ & $3000(1000-6000)$ & $U=2561$ & $0.011^{*}$ \\
\hline Intraoperative colloids (ml), median (range) & $500(450-1000)$ & $500(500-2000)$ & $U=822$ & $0.049^{*}$ \\
\hline Blood transfusion (units), median (range) & $2(1-6)$ & $2(1-7)$ & $U=1052$ & 0.161 \\
\hline FFP transfusion (units), median (range) & $2(2-4)$ & $4(2-6)$ & $U=13.5$ & 0.078 \\
\hline Platelet transfusion (units), median (range) & $6(3-8)$ & $3(2-4)$ & $U=2.5$ & 0.078 \\
\hline Estimated blood loss (ml), median (range) & $700(100-2500)$ & $1000(200-3000)$ & $U=2243$ & $0.001^{*}$ \\
\hline LOS (days), median (range) & $4(0.25-27)$ & $4(1-90)$ & $U=2796$ & 0.479 \\
\hline Discharge GCS, median (range) & $15(4-15)$ & $14(3-15)$ & $U=2286$ & 0.078 \\
\hline
\end{tabular}

${ }^{*} P<0.05$. INR=International normalised ratio, $\mathrm{SD}=$ Standard deviation, GCS=Glasgow Coma Scale, $n=$ Number of patients, $\mathrm{PT}=\mathrm{Prothrombin}$ time, APTT=Activated partial thromboplastin time, SGOT=Serum glutamic oxaloacetic transaminase, SGPT=Serum glutamic pyruvic transaminase, $\mathrm{FFP=Fresh}$ frozen plasma, LOS=Length of stay, CT=Computed tomography

Table 3: Change in the percentage of patients with coagulopathy from preoperatively to postoperatively

\begin{tabular}{lcccc}
\hline & Post-operative INR $<\mathbf{1 . 3}, \boldsymbol{n} \mathbf{( \% )}$ & Post-operative INR $>\mathbf{1 . 3}, \boldsymbol{n} \mathbf{( \% )}$ & Total & $\boldsymbol{P}$ \\
\hline Pre-operative INR $<1.3$ & $42(29.2)$ & $40(27.8)$ & 82 & 0.03 \\
Pre-operative INR $>1.3$ & $22(15.3)$ & $40(27.8)$ & 62 \\
Total & $64(44.4)$ & $80(55.6)$ & 144 \\
\hline
\end{tabular}

INR=International normalised ratio, $n=$ Number of patients 
They observed that coagulopathy was associated with both increased mortality (odds ratio: 9.0; 95\% CI: 7.3-11.6) and unfavourable outcome (odds ratio: 36.3; 95\% CI: 18.7-70.5). The incidence of early coagulopathy in another study by Wafaisade et al. ${ }^{[5]}$ was reported to be $23 \%$. Moreover, the frequency of this coagulopathy may continue to increase in the post-injury period. In a retrospective study by Greuters et al., in 107 patients, of which $65 \%$ had severe TBI, the authors reported the incidence of early coagulopathy to be $24 \% .{ }^{[6]}$ In the group of patients without early coagulopathy, $40 \%$ developed delayed coagulopathy. Hence, the overall incidence increased to $54 \%$ in the first $24 \mathrm{~h}$ and it had strong prognostic value for unfavourable outcome. The findings of our study also corroborate with the above literature.

Analysing the factors which may have causal association with coagulopathy, we found that pre-operative GCS was significantly lower in patients with coagulopathy. Keller et al. also reported that the risk of coagulopathy increased in patients with a lower GCS. ${ }^{[7]}$ Hence, coagulopathy may be a marker of the severity of head injury. A recent study also found that low GCS $<8$ or a high ISS (ISS $\geq 16$ ), hypotension on admission, and the presence of cerebral oedema, subarachnoid haemorrhage and midline shift are independent risk factors for developing coagulopathy in patients with isolated TBI. ${ }^{[3]}$

Traumatic injuries are a leading cause of morbidity and mortality, and coagulopathy is one of the potentially preventable factors. The usual common causes of coagulopathy in any traumatic injury are massive blood loss and subsequent fluid resuscitation leading to haemodilution. Significant contributors to coagulopathy are haemodilution from aggressive resuscitation, hypothermia, and acidosis, together known as the lethal triad. Colloids are highly effective at increasing the intravascular volume. Colloid resuscitation has been associated with a reduction in coagulation factors, platelet dysfunction and haemorrhagic complications. Clinical acidosis is commonly observed in trauma patients due to hypoperfusion from massive blood loss. Acidosis causes impaired clotting enzyme activities. As a proactive approach in damage control resuscitation, massive transfusion protocols quickly provide large amounts of blood products to critically injured and bleeding patients. However, these factors are less relevant in patients with isolated TBI.

Various treatment options for coagulopathy include administration of FFPs, antifibrinolytic agents, cryoprecipitate, recombinant factor VIIa (rFVIIa) and antithrombin III. It has been shown that treatment with rFVIIa within $4 \mathrm{~h}$ after the onset of intracerebral haemorrhage limits the expansion of the haematoma, reduces mortality and improves functional outcomes at 90 days. ${ }^{[8]}$ The most common cause postulated for the occurrence of coagulopathy in TBI is the release of tissue factor triggering intravascular coagulation. In addition, hyperfibrinolysis occurs which causes activation of protein $C$ and release of endothelial tissue plasminogen activator. In our study, all patients were normothermic and were haemodynamically stable, therefore, excluding hypothermia and acidosis as contributing causes of coagulopathy. However, we did not do arterial blood gas analysis in our patients.

In patients with coagulopathy after TBI, Harhangi et al. observed approximately ten times higher mortality and more than thirty times higher risk of poor outcomes compared to patients without coagulopathy. Recently, results from the IMPACT (International Mission for Prognosis and Clinical Trial) study showed that the PT is a powerful independent prognostic factor after TBI. ${ }^{[9]}$ We also found that coagulopathy was associated with increased blood loss leading to administration of more crystalloids and colloids. However, we did not find increased blood transfusion associated with blood loss probably because both blood loss as well as need for transfusion were assessed only clinically and no objective measures were used to guide transfusion. Although not statistically significant, patients with coagulopathy were more likely to receive FFPs and platelet transfusion in our study cohort. It was also observed that there was a trend towards lower discharge GCS in patients with coagulopathy. We did not find significantly poorer outcomes in patients with coagulopathy probably because, our sample size was not adequate.

The limitations of our study were, first, the estimation of blood loss and end point of transfusion were not objective. Second, the outcome of patients was studied only for a brief period till discharge. Third, we did not study the association of coagulopathy with imaging or arterial blood gas analysis. Finally, thromboelastography and thromboelastometry are tests of the point of care to assess entire clotting process and guide blood product therapy. This is the gold standard, but it is not available at our institute.

\section{CONCLUSION}

The incidence of coagulopathy in our study cohort which is a subpopulation of TBI patients presenting for surgery was $42.8 \%$ which is quite high. The severity of head injury as represented by GCS correlated with derangement of coagulation parameters. Coagulopathy leads to increased blood loss and increased intraoperative fluid and blood component requirements which can ultimately lead to increased morbidity and complications. Considering 
the prognostic implications of coagulopathy, we would like to emphasise the importance of monitoring of coagulation parameters at admission, with serial follow-ups as the haemostatic abnormalities may continue to worsen following the injury. ${ }^{[10]}$ In patients with low GCS, a high index of suspicion for deranged coagulation should be kept in mind.

\section{Acknowledgement}

We would like to thank Indian Society of Neuroanaesthesiology and Critical Care for providing funding for this study.

\section{Financial support and sponsorship}

From Indian Society of Neuroanaesthesiology and Critical Care for providing funding for this study.

\section{Conflicts of interest}

There are no conflicts of interest.

\section{REFERENCES}

1. Stein SC, Smith DH. Coagulopathy in traumatic brain injury. Neurocrit Care 2004;1:479-88.

2. HCarhangi BS, Kompanje EJ, Leebeek FW, Maas AI. Coagulation disorders after traumatic brain injury. Acta Neurochir (Wien)
2008;150:165-75.

3. Talving P, Benfield R, Hadjizacharia P, Inaba K, Chan LS, Demetriades D. Coagulopathy in severe traumatic brain injury: A prospective study. J Trauma 2009;66:55-61.

4. Epstein DS, Mitra B, Cameron PA, Fitzgerald M, Rosenfeld JV. Acute traumatic coagulopathy in the setting of isolated traumatic brain injury: Definition, incidence and outcomes. Br J Neurosurg 2015;29:118-22.

5. Wafaisade A, Lefering R, Tjardes T, Wutzler S, Simanski C, Paffrath $\mathrm{T}$, et al. Acute coagulopathy in isolated blunt traumatic brain injury. Neurocrit Care 2010;12:211-9.

6. Greuters S, van den Berg A, Franschman G, Viersen VA, Beishuizen A, Peerdeman SM, et al. Acute and delayed mild coagulopathy are related to outcome in patients with isolated traumatic brain injury. Crit Care 2011;15:R2.

7. Keller MS, Fendya DG, Weber TR. Glasgow coma scale predicts coagulopathy in pediatric trauma patients. Semin Pediatr Surg 2001;10:12-6.

8. Mayer SA, Brun NC, Begtrup K, Broderick J, Davis S, Diringer MN, et al. Recombinant activated factor VII for acute intracerebral hemorrhage. N Engl J Med 2005;352:777-85.

9. Murray GD, Butcher I, McHugh GS, Lu J, Mushkudiani NA, Maas AI, et al. Multivariable prognostic analysis in traumatic brain injury: Results from the IMPACT study. J Neurotrauma 2007;24:329-37.

10. Carrick MM, Tyroch AH, Youens CA, Handley T. Subsequent development of thrombocytopenia and coagulopathy in moderate and severe head injury: Support for serial laboratory examination. J Trauma 2005;58:725-9. 